\title{
RECHERCHES POLAROGRAPHIQUES SUR LES PROTÉINES DU LAIT
}

\author{
par \\ L. M. BURUIANA et V. PAVLU
}

La méthode d'analyse polarographique permet d'apprécier certains phénomènes de réduction qui se produisent à la cathode sous l'influence d'une tension électrique variable.

Le dispositif expérimental est constitué par une cellule d'électrolyse dont la cathode à gouttes de mercure est rapidement et complètement polarisable et dont l'anode est pratiquement non polarisable. Si la tension croit continuellement, on observe à un moment donné, un "saut" brusque de l'intensité suivie par une valeur constante, le courant limite. Le nombre des sauts correspondent à la réduction de l'hydrogène à l'électrode à gouttes de mercure.

Le phénomène enregistré photographiquement est représenté par un tracé d'aspect caractéristique et constitue le polarogramme ou la courbe polarographique de la solution à étudier.

Le potentiel de réduction et l'accroissement correspondant de l'intensité, sont des paramètres spécifiques pour une substance donnée.

J. Heyrovski, J. Babicka [1] et R. Brdicka [2] ont constaté que les protéines influencent spécifiquement la réduction polarographique d'une solution ammoniacale (de $p \mathrm{H}=9,5)$ de chlorure cobalteux ou cobaltique. Pour une concentration et un $p \mathrm{H}$ déterminé, on obtient une courbe dont l'aspect et les diverses particularités dépendent de la nature et de la concentration en protéines. Dans la figure 1 on a représenté le tracé polarographique de la solution de chlorure cobalteux étalon (solution de base, voir plus loin sa composition exacte) en présence (tracé 1) et en absence des protéines (tracé 2). On appelle un tel polarogramme, " courbe catalytique protéique", parce que c'est la protéine qui catalyse la réduction de l'hydrogène à la cathode pour une tension plus basse que de coutume.

D'après ce qu'on voit, l'effet catalytique de la protéine est caractérisé par la présence de deux sauts d'intensité appelés vagues ou marches protéiques, correspondant approximativement aux tensions de $-1,4$ volt et $-1,6$ volt, au lieu d'une seule vague pour $-1,8$ volt.

Cette observation des auteurs précités a constitué le point de départ de nombreuses recherches dans le domaine de la chimie des protéines. 
D'intéressants travaux ont été effectués par C. Tropp et ses collaborateurs, qui ont établi la corrélation entre l'aspect et les divers paramètres des courbes catalytiques protéiques et les propriétés des diverses protéines natives et dénaturées.

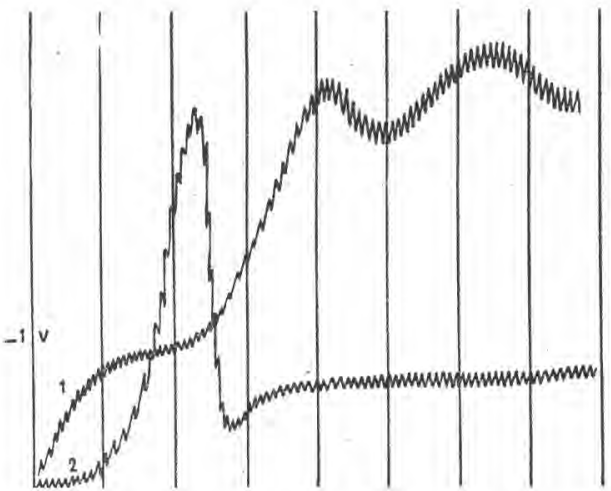

Fig. 1 .

Tracé 1. Solution de base à chlorure cobalteux.

Tampon ammoniacal $p \mathrm{H}=9,5$,

après adjonction d'une petite quantité de sérum de lait de vache.

Tracé 2. Même solution, sans sérum.

En ce qui concerne la cause de l'influence catalytique des protéines sur la réduction de l'hydrogène, l'opinion de la majorité des chercheurs est qu'elle est due à la présence et à l'arrangement de divers amino-acides contenus dans la molécule protéique, ce qui favorise la mobilité de l'hydrogène des groupements prototropiques. Surtout pour la deuxième vague (-1,6 volt) il est sûr qu'elle est due à la cystéine ou cystine, contenue par celle-ci.

La première vague (app. - 1,4 volt) serait due aux groupements prototropiques qui apparaissent après l'enchaînement des autres amino-acides contenus dans la molécule de protéine.

L a position et la hauteur des vagues polarographiques sont dues au courant de diffusion des protons dans "l'atmosphère " des gouttes de mercure à la cathode.

En dehors de ces phénomènes électroniques autour de la goutte de mercure, à la zone de séparation avec la solution, il se passe aussi des perturbations attribuées aux forces moléculaires.

Parmi celles-ci, un rôle important est joué par la tension superficielle. Des molécules tensioactives ajoutées à la solution de base peuvent modifier le degré de polarisation de l'électrode à gouttes de mercure. Par leur structure moléculaire complexe et par la présence de groupements fortement réactifs, les protéines modifient l'équilibre dynamique à la surface de la cathode mobile.

On appelle pouvoir supprimant (P.S.) la dilution nécessaire pour réduire de moitié l'intensité maximum de réduction de l'hy. drogène dans la solution de base. Cela s'appelle, dans le langage polarographique, réduire le maximum de cobalt à demi. Nous 
avons exprimé cette dilution par la quantité, en milligramme protéine, contenue dans 100 millilitres de solution de base.

La détermination du pouvoir supprimant se fait par tatonnement, car il n'y a pas proportionnalité entre celui-ci et la quantité de substance active.

L'aspect de la courbe catalytique protéique, le nombre et la hauteur des vagues, le pouvoir supprimant, etc., sont des paramètres qui caractérisent les diverses protéines. Avec l'électrophorèse, l'ultracentrifugation, l'analyse spectrale en ultra-violet et infra-rouge, l'analyse polarographique peut compléter les informations relatives à la micro et macrostructure des protéines. Elle peut même servir comme méthode très sensible pour l'identification des substances protéiques dans les éluats chromatographiques, comme l'a préconisé B. Drake [3].

Le but du présent travail est de faire connaître le comportement polarographique des diverses protéines du lait, de signaler et de suggérer des possibilités éventuelles pour son contrôle hygiénique et industriel.

A part l'électrophorèse, rares sont les techniques qui recourent à l'analyse structurale des protéines du lait pour connaître l'influence des facteurs comme l'alimentation, les maladies, l'effet de l'amélioration, etc.

En ce qui concerne l'analyse polarographique des protéines du lait, on connaît peu de travaux dans ce domaine. T. GAL [4] a fait les premières observations, en remarquant l'action empêchante de la caséine et l'influence de divers facteurs sur les protéines du sérum.

\section{A. Comportement polarographique du lait}

Parce que la matière grasse du lait souille l'électrode à gouttes de mercure, il est besoin de la dégraisser au préalable. Une centrifugation de 10-15 minutes à 5.000 tours/minute suffit pour obtenir un lait qui n'empêche plus les enregistrements polarographiques.

Les mesures ont été faites à l'aide d'un polarographe de type Heyrovski V301 avec une cellule ouverte de Spalenka. Le temps d'égouttage du mercure à la cathode était de trois secondes. La température des enregistrements était de $25^{\circ} \mathrm{C}$. La tension sur le tambour potentiométrique $-1,9$ volt. On a toujours utilisé $1 / 100^{\mathrm{e}}$ de la sensibilité maximum du galvanomètre, qui est de l'ordre de $10^{-8} \mathrm{amp}$. pour un déplacement du spot de 1 millimètre à 1 mètre distance.

Dans le cas des déterminations du pouvoir supprimant on a employé la sensibilité de $1 / 300^{e}$. 
La solution de base à chlorure cobalteux avait la composition suivante :

$2 \mathrm{ml} . \mathrm{CoCl}_{2} 1,6 \cdot 10^{-2} \mathrm{M}+1 \mathrm{ml}^{2} \mathrm{NH}_{4} \mathrm{Cl} \mathrm{M}+1 \mathrm{ml} \cdot \mathrm{NH}_{4} \mathrm{OH} \mathrm{M}+6 \mathrm{ml}$. eau distillée. A 2 millilitres de cette solution, on ajoutait très exactement, à l'aide d'une micropipette à vis, des quantités variables de lait. On a calculé finalement la concentration en milligrammes de protéine pour cent. Les concentrations étaient choisies par des tatonnements préalables de manière à bien préciser le point de croisement, ou point d'inversion de Tropp.

C. TRopp [5] a observé pour la première fois que, en diminuant la concentration des solutions protéiques soumises à l'analyse polarographique, il arrivait, dans le cas des protéines d'origine animale [6], à un moment donné, que la hauteur de la deuxième vague (vague de la cystéine) devenait plus basse que la première. Tropp appela ce phénomène "effet de croisement "(Kreuzungseffekt). Nous utiliserons dans le cours de ce travail l'expression de point d'inversion (P. I.) pour la concentration en milligrammes pour cent de protéine de la solution, diluée convenablement, pour que la deuxième vague soit plus basse que la première. Le $P$. I. constitue un paramètre qui pourrait caractériser les diverses protéines soumises à l'analyse polarographique.

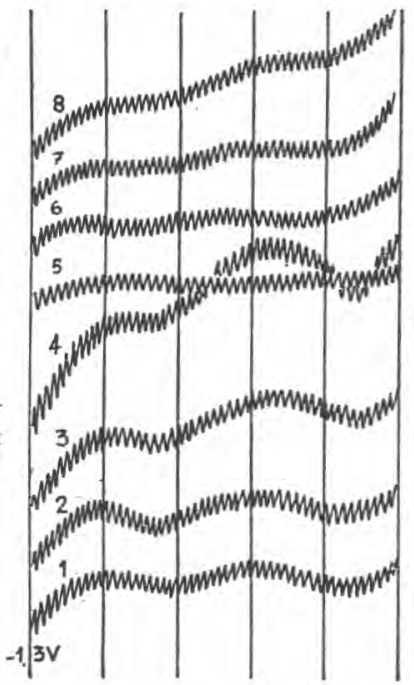

Fig. 2.

Polarogramme du lait de vache frais, non cuit.

Tracé 1. Concentration en protéines $1 \mathrm{mgr} .58 \%$.

Tracé 2. Concentration en protéines $2 \times 1 \mathrm{mgr} .58 \%$.

Tracé 3. Concentration en protéines

$$
4 \times 1 \mathrm{mgr} .58 \% \text {. }
$$

Tracé 4. Concentration en protéines

$$
8 \times 1 \mathrm{mgr} .58 \% \text {. }
$$

Point d'inversion (P. I.) $=1 \mathrm{mgr} .58 \%$.

Pouvoir supprimant (P. S.) $=14 \mathrm{mgr} .4 \%$.

Tracés 5, 6, 7, 8, le même lait, bouilli.

Pouvoir supprimant après chauffage (P. S.) $=25 \mathrm{mgr} .75 \%$.

Pour comparer l'aspect des courbes polarographiques et l'influence de la concentration en protéines, nous représenterons dans les figures suivantes une "famille" de courbes catalytiques, obtenues avec des concentrations croissantes en progression géomé- 
trique. Dans la figure 2, on voit les courbes polarographiques pour un lait de vache frais non cuit (tracés $1,2,3,4$ ). On distingue l'effet Tropp pour le tracé 1 . Le point d'inversion (P. I.) correspond à la concentration de $1 \mathrm{mgr}$. $58 \%$ de protéines totales.

Pour voir quelle était l'action de la chaleur, on a chauffé le lait à l'ébullition maintenue pendant 5 minutes, dans un bainmarie. Les courbes catalytiques protéiques sont représentées dans la même figure 2 par les tracés $5,6,7,8$. On remarque l'abaissement sensible de la hauteur des vagues. Le P. I. reste le même, mais le P. S. croit beaucoup ( 2 mgr. $57 \%$, au lieu de $1 \mathrm{mgr} .44 \%$ dans le cas du lait non chauffé).

L'influence de l'urée, en concentration à $30 \%$, sur le lait, a été enregistrée par le polarogramme de la figure 3 .

Fig. 3.

Effet de l'urée sur le comportement polarographique du lait frais, non cuit.

Tracés $1,2,3,4$, sans urée P. S. $=23 \mathrm{mgr} .65 \%$.

Tracés 5, 6, 7, 8, avec $30 \%$ d'urée P. S. $=40 \mathrm{mgr} .05 \%$.

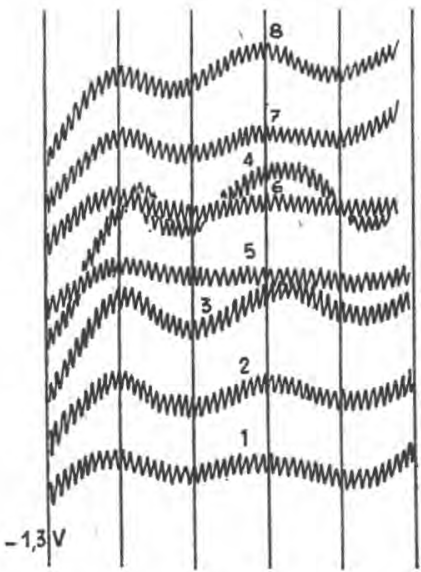

On remarque que l'urée, de même que l'échauffement, abaissent les vagues polarographiques. La dénaturation des protéines par l'urée a fait l'objet de nombreuses recherches depuis la première observation de Hopkins [7]. D'après Mirsky et Pauling [8] l'effet dénaturant de l'urée est attribué à la rupture des liaisons d'hydrogène qui réunissaient les chaines polypeptidiques de la molécule protéique. Ultérieurement, il se forme de nouvelles liaisons d'hydrogène, cette fois entre le dénaturant et les chaines polypeptidiques, avec l'apparition d'un édifice en nid d'abeille plus rigide, ce qui réduit la mobilité des protons et en conséquence, leur énergie. Du point de vue polarographique, cela se traduit par l'abaissement de la hauteur des vagues de la courbe catalytique protéique. Les nouvelles molécules dénaturées sont moins tensioactives à cause de leur structure, ce qui provoque l'accroissement de la valeur de P. S. On peut donc expliquer par les considérations 
faites ci-dessus l'effet de l'urée sur le comportement des courbes polarographiques.

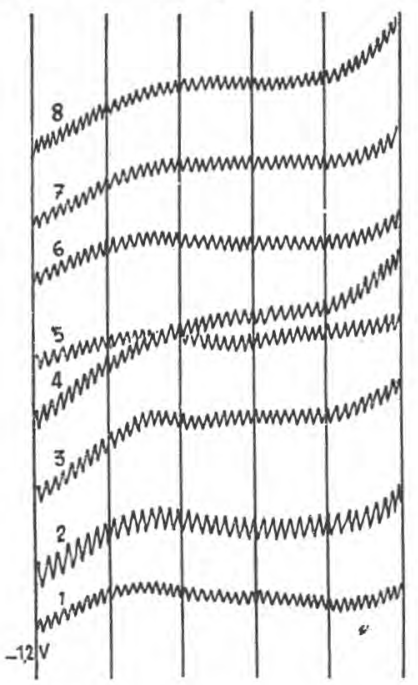

Fig. 4.

Polarogramme du lait de femme.

Tracé 1. Concentration en protéines $4 \mathrm{mgr}$. \%.

Tracé 2. Concentration en protéines

$$
2 \times 4 \mathrm{mgr} \text {. \%. }
$$

Tracé 3. Concentration en protéines

$$
4 \times 4 \mathrm{mgr} \text {. \%. }
$$

Tracé 4. Concentrarion en protéines

$$
8 \times 4 \mathrm{mgr} \text {. \%. }
$$

P. I. $=8 \mathrm{mgr} . \%-$ P. S. $=16 \mathrm{mgr} .0 \%$.

Tracés 5, 6, 7, 8, le même lait, bouilli.

P. I. $=8 \mathrm{mgr} . \%-$ P. S. $=16 \mathrm{mgr} .0 \%$.

Nos recherches ont montré que, pour des laits normaux, l'aspect des courbes catalytiques, la hauteur des vagues, le P. I. et le P. S. oscillaient entre des limites très étroites.

\section{Fig. 5.}

Polarogramme du lait de femme (autre échantillon).

Tracé 1. Concentration en protéines

$$
4 \mathrm{mgr} .84 \% \text {. }
$$

Tracé 2. Concentration en protéines

$$
2 \times 4 \text { mgr. } 84 \% \text {. }
$$

Tracé 3. Concentration en protéines

$$
4 \times 4 \mathrm{mgr} .84 \% \text {. }
$$

Tracé 4. Concentration en protéines

$$
8 \times 4 \mathrm{mgr} .84 \% \text {. }
$$

P.I. $=19 \mathrm{mgr} .36 \%-\mathrm{P} . \mathrm{S} .=19 \mathrm{mgr} .35 \%$.

Tracés 5, 6, 7, 8, le même lait, bouilli.

P. I. $=19$ mgr. $36 \%$ P. S. $=19$ mgr. $35 \%$

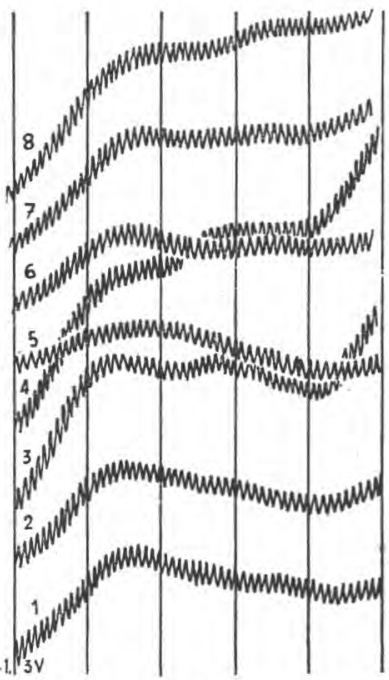

La dilution, l'infection de la mamelle surtout, l'époque de la lactation, etc., influencent sensiblement les paramètres polarographiques. L'influence des divers facteurs et les limites de variation sur les divers paramètres, seront publiés en une autre occasion, 
ce qui permettra de préciser la valeur de cette méthode pour le contrôle hygiénique et industriel du lait.

LAIT DE FEMME. - L'aspect des courbes polarographiques dans le cas du lait de femme est bien différent.

Il y manque quelquefois, presque complètement, la deuxième vague caractéristique des courbes catalytiques protéiques (figure 4).

Dans un autre cas, l'aspect de la courbe est un peu différent. Il est vrai que la concentration en protéines totales est plus grande (figure 5).

La valeur de P. I. est douze fois plus grande que pour le lait de vache. Le fait est d'autant plus remarquable que le contenu en caséine du lait de femme est plus petit que celui du lait de vache. On verrait même, que la caséine exerce une action inhibitrice sur les phénomènes de réduction des protons à la cathode.

\section{B. Comportement polarographique des protéines du sérum du lait}

Par précipitation avec de l'acide acétique à $p \mathrm{H}=4,8$, la caséine du lait de vache précipite. Le sérum obtenu contient entre 0,5-0,9\% de matières protéiques (approximativement 20\% des protéines totales). Les protéines du sérum sont constituées par les trois fractions suivantes : lactalbumine, lactoglobuline (de Palmer) et globuline [9]. De coutume, deux fractions principales se séparent par précipitation fractionnée à l'aide de sulfate d'ammonium : les globulines, ou mieux les lactoglobulines (à 0,5 de saturation), et les lactalbumines (à saturation totale) [10]. Nous avons recouru à ce procédé pour séparer les protéines du sérum.

\section{Fig. 6 .}

Polarogramme du sérum de lait de vache.

Tracé 1. Concentration en protéines 0 mgr. $137 \%$.

Tracé 2. Concentration en protéines $2 \times 0 \mathrm{mgr} .137 \%$.

Tracé 3. Concentration en protéines $4 \times 0 \mathrm{mgr} .137 \%$.

Tracé 4. Concentration en protéines $8 \times 0 \mathrm{mgr} .137 \%$. P. I. $=0 \mathrm{mgr} .137 \%-$ P.S. $=19 \mathrm{mgr} .40 \%$. Tracés $5,6,7,8$, le même sérum, bouilli.

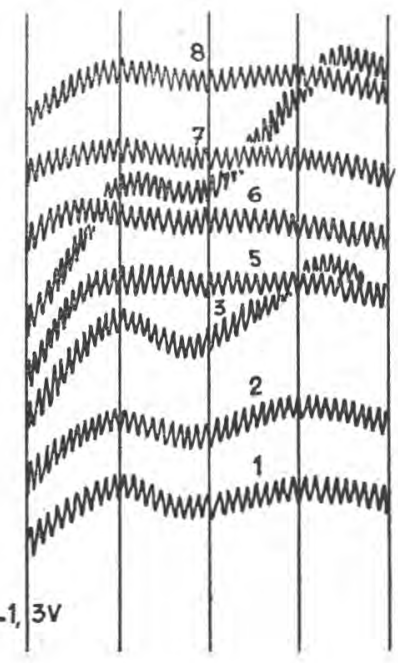




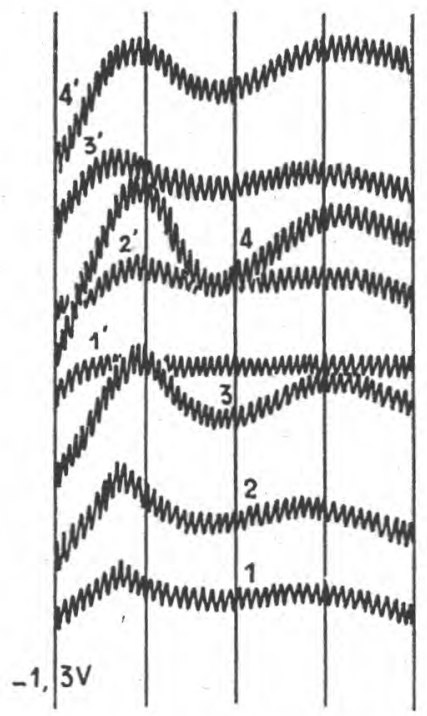

Fig. 7.

Polarogramme du sérum de lait de vache (II).

Tracé 1. Concentration en protéines

$$
0 \text { mgr. } 084 \% \text {. }
$$

Tracé 2. Concentration en protéines

$$
2 \times 0 \text { mgr. } 084 \% \text {. }
$$

Tracé 3. Concentration en protéines

$$
4 \times 0 \text { mgr. } 084 \% \text {. }
$$

Tracé 4. Concentration en protéines

$$
8 \times 0 \mathrm{mgr} .084 \% \text {. }
$$

P. I. $=1 \mathrm{mgr} .34 \%-$ P. S. $=11 \mathrm{mgr} .95 \%$.

Tracés 5, 6, 7, 8, le même lait, bouilli.

Dans la figure 6 sont représentées les courbes catalytiques protéiques du sérum acétique.

Fig. 8.

Polarogramme du même sérum (fig. 7) après dialyse.

Tracé 1. Concentration en protéines $0 \mathrm{mgr} .075 \%$.

Tracé 2. Concentration en protéines

$$
2 \times 0 \mathrm{mgr} .075 \% \text {. }
$$

Tracé 3 . Concentration en protéines

$$
4 \times 0 \mathrm{mgr} .075 \% \text {. }
$$

Tracé 4. Concentration en protéines

$$
8 \times 0 \mathrm{mgr}, 075 \% \text {. }
$$

P. I. $=0$ mgr. $3 \%-$ P. S. $=5$ mgr. $50 \%$.

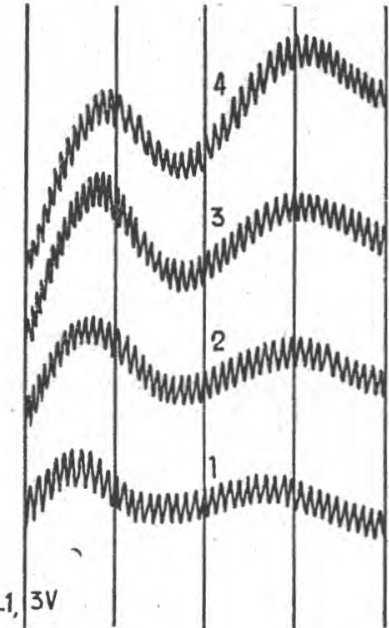

En général, pour divers échantillons de lait, l'aspect et les valeurs de P. I. et P. S. oscillent très peu.

Quelquefois, on rencontre un comportement différent (figure 7).

L'aspect et la hauteur des vagues peuvent être influencés par d'autres substances existant dans le sérum; aussi, l'avons nous soumis à la dialyse. 
Monnier et Besso [11], étudiant la salive au point de vue polarographique, ont obtenu une courbe catalytique caractérisée par trois vagues dont deux, correspondant aux tensions $\mathrm{E}_{\frac{1}{2}}-1,60$ volt et $\mathrm{E} \frac{1}{2}-1,75$ volt, disparaissaient par dialyse en se retrouvant dans le dialysat.

En dialysant le sérum, nous avons obtenu les courbes catalytiques représentées dans la figure 7 .

On remarque que le point d'inversion (P. I.) a diminué, de $1 \mathrm{mgr} .34 \%$ à $0 \mathrm{mgr} .3 \%$ et le P. S. de $2 \mathrm{mgr} .99 \%$ à $1 \mathrm{mgr} .38 \%$.

Pour, les laits d'aspect polarographique normal, la dialyse n'a eu aucun effet, ou très peu.

On peut conclure que dans certains laits, il existe des substances dialysables qui peuvent influencer la structure macromoléculaire des protéines sériques et donc leur comportement polarographique. Le fait mérite d'être étudié avec attention.

Effet de L'acidification Naturelle du lait. - Pendant. l'acidification prolongée du lait, nous avons obtenu une modification radicale du comportement polarographique des protéines sériques.

Le fait est représenté dans la figure 9 .

Fig. 9.

Polarogramme du sérum de lait acidifié par fermentation lactique prolongée

(80 heures)

Tracés 1, 2, 3, 4, sérum non bouilli.

P. S. $=19 \mathrm{mgr} .5 \%$.

Tracés 5, 6, 7, 8, le même sérum, bouilli.

P. S. $=27 \mathrm{mgr} .0 \%$.

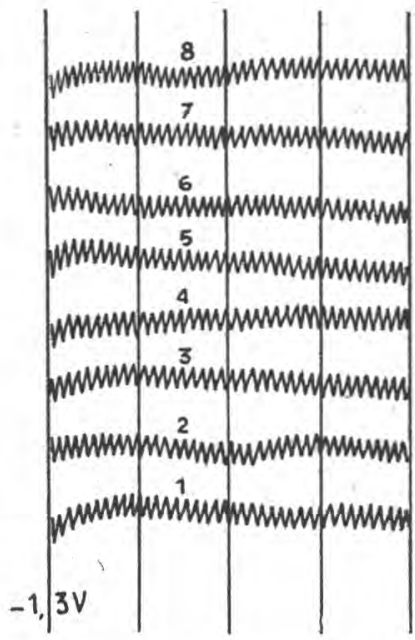

L'altération profonde des protéines sériques sous l'action des bactéries lactiques est très visible. Il faut mentionner que le sérum donnait toutes les réactions des protéines natives et précipitait abondamment par chauffage.

Pour étudier séparément les propriétés polarographiques de la l actoglobuline et de la lactalbumine, nous les avons. isolées par fractionnement au sulfate d'ammonium. 


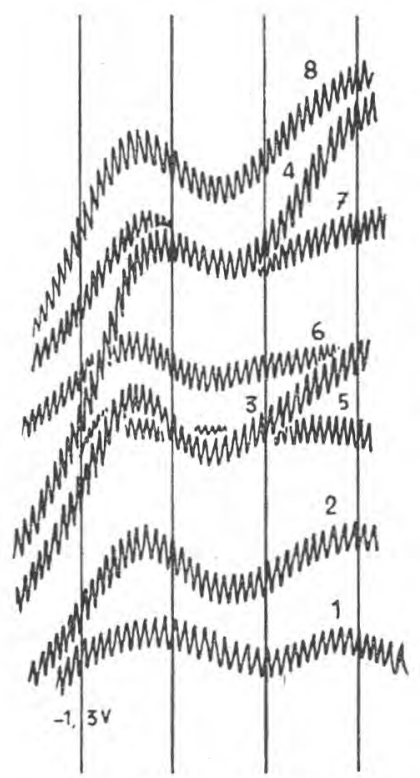

Fig. 10.

Polarogramme d'une solution de lactalbumine

Tracé 1. Concentration en lactalbumine $4 \mathrm{mgr} .72 \%$.

Tracé 2. Concentration en lactalbumine $2 \times 4 \mathrm{mgr} .72 \%$.

Tracé 3 . Concentration en lactalbumine $4 \times 4 \mathrm{mgr} .72 \%$.

Tracé 4. Concentration en lactalbumine $8 \times 4$ mgr. $72 \%$.

P. I. $=4 \mathrm{mgr} .72 \%-$ P. S. $=30 \mathrm{mgr} .1 \%$.

Tracés 5, 6, 7, 8, la même solution, dénaturée avec de l'urée à $30 \%$.

P. S. $=94 \mathrm{mgr} .4 \%$.

Les deux fractions ont été ensuite dialysées jusqu'à la disparition de la réaction de l'ion ammonium. Pour la lactoglobuline, on termine la dialyse contre une solution à $0,7 \%$ de chlorure de sodium. Dans la figure 10 sont enregistrées les courbes catalytiques obtenues avec la solution de lactalbumine.

Fig. 11.

Polarogramme d'une solution de lactoglobuline.

Tracé 5. Concentration en lactoglobuline $3 \mathrm{mgr} .12 \%$.

Tracé 6. Concentration en lactoglobuline $2 \times 3 \mathrm{mgr} .12 \%$.

Tracé 7. Concentration en lactoglobuline $4 \times 3 \mathrm{mgr} .12 \%$.

Tracé 8. Concentration en lactoglobuline $8 \times 3 \mathrm{mgr} .12 \%$.

P. I. $=55$ mgr. $6 \%-$ P. S. $=77 \mathrm{mgr} .5 \%$.

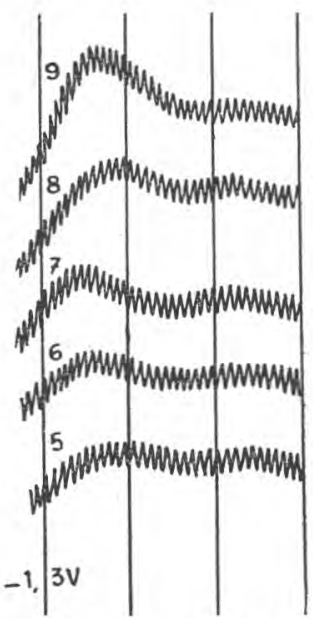

Si nous comparons les valeurs de P.I. et P.S. de la lactalbumine séparée du même sérum et dialysée avec les P. I. et P. S. du sérum, on constate une différence appréciable (le P. I. du sérum était $0 \mathrm{mgr}$. $127 \%$ et le P. S. $=3 \mathrm{mgr} .5 \%$ ). Il est vrai que le sérum 
contenait aussi la lactoglobuline, mais celle-ci influence plutôt négativement le P. I. et le P.S.

Il résulte de cette constatation que la précipitation avec le sulfate d'ammonium, s'il est réversible du point de vue de la solubilité, dénature profondément la macrostructure de la lactalbumine, fait souvent négligé.

Dans le cas de la lactoglobuline, la courbe catalytique a l'aspect enregistré dans la figure 11.

On remarque que le $\mathrm{P}$. I. et le P.S. sont, de beaucoup, plus grands pour la lactoglobuline que pour la lactalbumine, ce qui dénote une plus petite activité polarographique de cette protéine.

Les valeurs trouvées par nous diffèrent de celles rapportées par C. Tropp (loc. cit.) qui indique pour les globulines du sérum sanguin un P. I. = 20 milligrammes $\%$ et pour limite de sensibilité, $1 \mathrm{mgr} .2 \%$ (dans notre cas, pour la lactoglobuline : $3 \mathrm{mgr} .12 \%$ ). On peut attribuer cette différence à la dénaturation par le sulfate d'ammonium.

Le rapport entre les hauteurs des vagues $h_{1} / h_{\iota}$ est, de beaucoup, plus grand pour la lactoglobuline que pour la lactalbumine.

En tenant compte que la hauteur $h_{2}$ de la deuxième vague est proportionnelle au contenu en cystéine et cystine des protéines et que la lactoglobuline contient plus de ces thioamino-acides $(3,5 \%$ d'après HaWk, Oser et Summerson) que la lactalbumine $(2,95$ d'après PALLADIN) cela signifie que l'activité polarographique doit être attribuée non pas à la quantité des amino-acides mais à leur manière d'enchainement dans l'édifice macromoléculaire de chaque protéine respective.

D'après les recherches de Trout et Weinstein [12], les protéines du sérum du lait de vache ont une forte influence sur l'oxydabilité des graisses du lait. En plus la quantité de la globuline serait proportionnelle à la production laitière des animaux [13]. Tenant compte de la différence constatée entre ces deux protéines du point de vue polarographique, on pourra utiliser cette méthode dans les diverses recherches concernant la sélection et la production animales.

L'effet dénaturant de l'urée sur la lactalbumine peut être observé dans la figure 10 (tracés $5,6,7,8$ ). La hauteur des vagues est abaissée et le P. S. fortement accru (de $30 \mathrm{mgr}$. $1 \%$ à $94 \mathrm{mgr} .4 \%$ ).

\section{Comportement polarographique de la caséine}

On sépare cette protéine du lait, préalablement dégraissé par centrifugation, à l'aide de l'acide acétique (à $p \mathrm{H}=4,8$ ).

On lave à fond avec de l'eau distillée le précipité sur le filtre 
et ensuite on le détache et on le solubilise dans un volume égal à celui du lait, d'une solution tampon de phosphates $\mathrm{M} / 15$ de $p \mathrm{H}=6,8$ celui du lait, d'une solution tampon de phosphates $\mathrm{M} / 15$ de $p \mathrm{H}=$ 6,8. Les courbes polarographiques pour des concentrations très larges sont représentées dans les figures 12 et 13 .

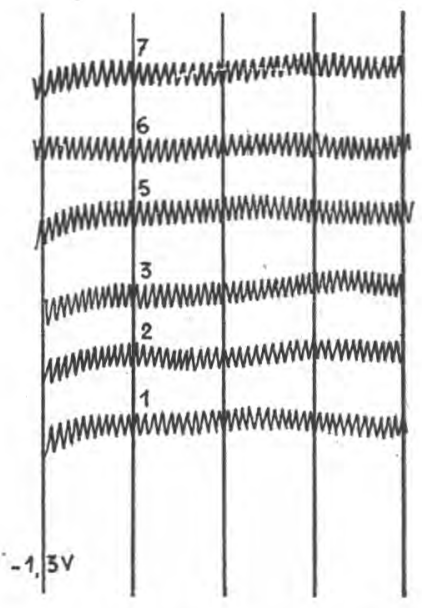

Fig. 12.

Polarogramme de la caséine (I).

Trace 1. Concentration en caséine

$1 \mathrm{mgr} .1 \%$.

Trace 2. Concentration en caséine

$2 \times 1 \mathrm{mgr} .1 \%$.

Trace 3. Concentration en caséine

$$
\begin{aligned}
& 4 \times 1 \mathrm{mgr} .1 \% \text {. } \\
& \text { P. S. }=28 \mathrm{mgr} . \% .
\end{aligned}
$$

Tracés $5,6,7$, les mêmes, après dénaturation avec de l'urée à $30 \%$.

P. S. $=44 \mathrm{mgr} \%$,

On constate que même pour des concentrations assez larges, la caséine ne catalyse pas la réduction de l'hydrogène à la cathode à gouttes de mercure. Le fait peut être expliqué par la composition chimique de la caséine et la structure macromoléculaire qui en résulte. Il est certain que les radicaux phosphoriques contenus dans la molécule de caséine jouent un rôle important quant à cette particularité polarographique. L'existence d'une couche "protectrice» fait soutenu en une autre occasion [14], peut expliquer aussi l'inertie de la caséine envers le niveau énergétique des protons, donc sur leur mobilité.

Fig. 13.

Polarogramme de la caséine (II).

Tracé 1. Concentration en caséine 25 mgr. $\% 5$

Tracé 2. Concentration en caséine

$$
2 \times 25 \mathrm{mgr} . \% 5
$$

Tracé 3. Concentration en caséine

$$
4 \times 2 \text { à mgr. } \% 5
$$

Tracé 4. Concentration en caséine

$$
8 \times 2 \text { à mgr. } \% 5
$$

Tracé 5. Concentration en caséine P. S. $=29 \mathrm{mgr} . \%$.

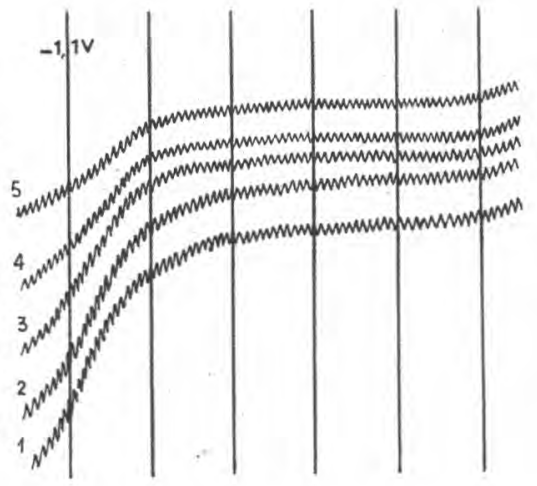


Sous l'influence de la trypsine, les solutions de caséine deviennent polarographiquement actives. Nous avons soumis à digestion, pendant dix minutes à $37^{\circ}, 20$ millilitres de la solution précédente de caséine, avec 5 millilitres d'extrait pancréatique d'activité trypsinique de 300 unités/millilitre, d'après NORTHRoP [15].

Les résultats sont enregistrés dans la figure 14 .

$$
\text { Fig. } 14 .
$$

Polarogramme de la caséine après digestion avec de la trypsine (10 minutes).

Tracé 2. Concentration en protéines

$11 \mathrm{mgr} .3 \%$.

Tracé 3. Concentration en protéines

$$
2 \times 11 \mathrm{mgr} .3 \% \text {. }
$$

Tracé 4. Concentration en protéines

$$
4 \times 11 \text { mgr. } 3 \% \text {. }
$$

Tracé 5. Concentration en protéines

$$
8 \times 11 \mathrm{mgr} .3 \% \text {. }
$$

\section{P. S. $=19 \mathrm{mgr} .65 \%$.}

La solution de caséine était bouillie après digestion.

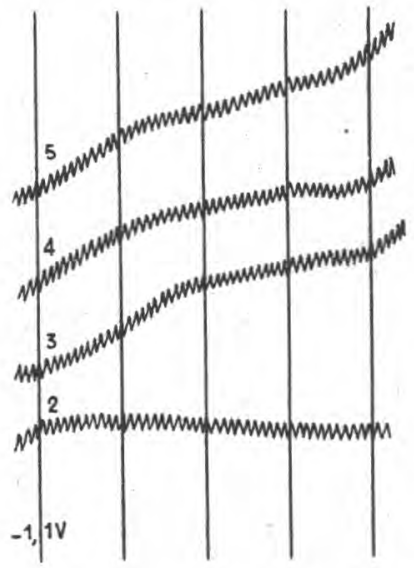

On remarque la similitude des courbes catalytiques de la caséine digérée avec celles du lait bouilli (figures 2, 3, ..).

Si on continue la digestion par la trypsine (jusqu'à 60 minutes), on constate l'apparition encore plus nette des vagues catalytiques protéiques (figure 15).

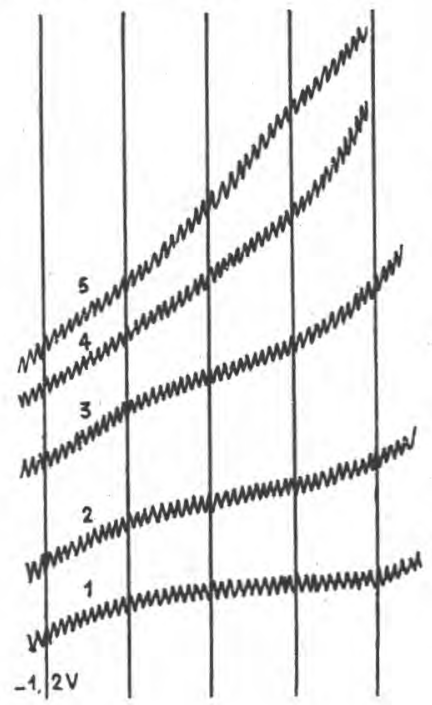

Fig. 15.

Polarogramme de la caséine après digestion prolongée (60 minutes) avec de la trypsine.

Tracé 1. Concentration en protéines

$$
5 \mathrm{mgr} .68 \% \text {. }
$$

Tracé 2. Concentration en protéines

$$
2 \times 5 \mathrm{mgr} .68 \% \text {. }
$$

Tracé 3. Concentration en protéines

$$
4 \times 5 \mathrm{mgr} .68 \% \text {. }
$$

Tracé 4. Concentration en protéines

$$
8 \times 5 \mathrm{mgr} .68 \% \text {. }
$$

Tracé 5. Concentration en protéines

$$
16 \times 5 \mathrm{mgr} .68 \% \text {. }
$$

P. S. $=11$ mgr. $35 \%$.

La solution de caséine était bouillie après digestion. 
Quant à la hauteur plus réduite de la deuxième vague, le fait peut être expliqué par la teneur réduite de la caséine en cystéine (seulement $0,3 \%$ ), dix fois plus petite que celle des autres protéines du lait. On remarque aussi la valeur plus petite du P. S. après digestion (11 mgr. $35 \%$ au lieu de $29 \mathrm{mgr} . \%$ ) ce qui dénote que la trypsine a détruit l'édifice macromoléculaire de la caséine en libérant les groupements actifs du point de vue polarographique.

Nous voyons aussi l'importance de cette méthode pour suivre l'action de diverses enzymes sur la structure des protéines.

\section{Conclusions}

On a appliqué la méthode d'analyse polarographique pour la. recherche des propriétés spécifiques des diverses protéines du lait :

$1^{\circ}$ Le lait de vache se comporte du point de vue polarographique comme une solution protéique, en donnant des courbes catalytiques caractéristiques avec la solution étalon de chlorure cobalteux.

Les deux vagues correspondent à $-1,40$ volt et $-1,60$ volt, les hauteurs $h_{1}$ et $h_{2}$ variant avec la quantité de lactalbumine et de lactoglobuline et avec leur rapport.

$2^{\circ}$ Par chauffage, la hauteur des vagues s'abaisse. L'urée a le même effet.

$3^{\circ}$ Il existe une différence nette entre le comportement polarographique du lait de vache et celui du lait de femme. Entre autres le chauffage n'influence ni la hauteur des vagues ni le P. S. (pouvoir supprimant) de celui-ci.

$4^{\circ}$ L'activité polarographique du lait est due à la lactalbumine et à la lactoglobuline du lait, la caséine étant inactive.

$5^{\circ}$ Après fractionnement à l'aide du sulfate d'ammonium, on a étudié séparément la lactalbumine et la lactoglobuline. Il y a une grande différence entre le comportement polarographique de ces deux protéines du lait. La plus active est la lactalbumine.

$6^{\circ}$ Après précipitation et dialyse, des protéines du sérum du lait, on constate, à l'aide de la méthode polarographique, que l'édifice moléculaire a été profondément changé.

$7^{\circ}$ L'urée et la chaleur modifient le mode de comportement des protéines du sérum; de même, la fermentation lactique.

$8^{\circ}$ La caséine est inactive du point de vue polarographique. Cela peut s'expliquer par sa structure macromoléculaire spéciale, caractérisée par la présence de groupements phosphoriques. A la suite de l'action protéolytique de la trypsine, la caséine devient polarographiquement active. Les courbes catalytiques de la caséine protéolysée ressemblent à celles du lait bouilli.

90 La méthode d'analyse polarographique donne des informations précieuses sur les protéines du lait. Elle pourrait être employée 
pour le contrôle hygiénique et industriel, mais surtout pour saisir les fines modifications de la macrostructure des protéines, sous l'influence des divers facteurs comme l'alimentation, les maladies, les facteurs climatiques et la sélection.

\title{
BIBLIOGRAPHIE
}

[1] J. Heyrovski et J. Babicka. Coll. des Travaux Chim. de Tchécoslovaquie. 1930, 2, 270.

[2] R. Brdicka. Coll. des Travaux Chim. de Tchécoslovaquie. 1933, 5, 112.

[3] B. Drake. Acta Chim. Skand. 1950, 5, 152.

[4] T. GAL. Prumysl Potravin. 1954, 5, 152, 206. Ref. Chem. Abst. 1954, 48, $13471 \mathrm{i}$.

[5] C. Tropp, L. Jühling und F. Geiger. Z. Physiol. Chem. 1939-1940, $262,225$.

[6] C. Tropp, F. Getger und W. Stoje. Z. Physiol. Chem. 1943, 277, 192.

[7] F. G. Hopkins. Nature. 1930, 126, 328.

[8] A. E. Mrrsky and L. Pauling. Proc. Natl. Acad. Sci. U.S. 1936, 22, 439 .

[9] Th. L. MoMeenin and B. D. Polis. Milk Proteins. In Advances in Protein Chemistry. 1949, 5. Acad. Press. Inc. Publishers. New-York, 201.

[10] E. L. Sмith. Journal Biol. Chem. 1946, 165, 665.

[11] D. Monnier et Z. Besso. Helv. Chim. Acta. 1954, 37, 455.

[12] G. M. Trout and B. R. Weinstein. XIII Internat. Dairy Congress, La Haye, 1953, 3, 1061.

[13] E. Pijanowski, B. Habaj et B. Hyziak. Bull. de l'Acad. Polonaise de Sci. 1956, 4, 11, 383.

[14] L. M. Buruiana et A. Fostiropol. Le Lait. 1956, 36, noo 359-360, 593.

[15] J. H. Northrop and M. Konitz. J. Gen. Physiol. 1953, 16, 313.

\section{L'ÉVOLUTION DE LA PRODUCTION LAITIËRE EN FRANCE (1)}

\author{
par \\ JACQUES CASALIS \\ Ingénieur Agricole
}

Professeur à l'Ecole Nationale des Industries Agricoles et Alimentaires

Les statistiques montrent qu'en moins d'un siècle les quantités de lait annuellement produites sur le territoire français ont presque triplé. Certes, on peut faire quelques réserves sur la valeur absolue des chiffres du tableau I qui sont pourtant extraits de documents officiels. (Ainsi, par exemple, suivant les auteurs et les méthodes de travail, les évaluations pour 1954 oscillent entre 180 et 220 millions d'hectolitres.)

(1) Le Paysan, 1957, ro 470. 